\section{DID PAEDIATRIC EARLY WARNING SYSTEM(PEWS) UTILISATION IMPACT ON SEVERITY OF ILLNESS AND OUTCOME FOR PATIENTS ADMITTED TO A TERTIARY PICU?}

\author{
G. Sefton, C. McGrath \\ PICU, Alder Hey Childrens NHS foundation Trust, \\ Liverpool, UK
}

Introduction: PEWs was implemented at Alder Hey in August 2006. No screening tool was being used at District General Hospitals at that time. There has been no comparative data to date exploring the impact of such innovations on the severity of illness at first contact and their outcomes.Method: Data is prospectively collected on all children admitted to PICU as part of a national dataset. Data was analysed for a year before and a year following PEWs implementation comparing all unplanned admissions from wards within the hospital with external unplanned admissions transferred from DGHs.

Results: There was no significant difference to the absolute numbers of patients admitted as emergency from wards $14.4-15.9 \%$ within the hospital or external sources $28.9-30.7 \%$.

For the internal unplanned admissions from wards in the hospital:

Median PIM scores reduced from 0.060 to 0.048

Inotrope requirement reduced from 32.5 to $23.4 \%$ of patient group,

Invasive ventilation requirement reduced from $75.1 \%$ to $62 \%$ of patient group, duration reduced from 6 to 4 days

Median length of stay reduced from 4.5 to 3 days.

For the unplanned admissions transferred from other hospitals

Median PIM scores increased from 0.057 to 0.071

95-97\% of children were invasively ventilated for transfer but median duration of ventilation was 3 days

Inotrope requirement was similar 29.8-31.8\%

Median duration of stay increased from 2 to 3 days

Mortality for both groups was similar 9-10.8\%
Implications: The wider use of PEWs at DGHs could reduce severity of illness and inotrope requirement of critically ill children

\section{5}

\section{MORTALITY RISK SCORES FOR VERY-LOW- BIRTHWEIGHT INFANTS (VLBWI) ADMITTED TO A SINGLE NEONATAL UNIT (NICU) IN A 15 YEAR PERIOD}
A. Rodríguez, I. Saez de Ugarte, E. Guerra, A. Azpeitia, A. Valls i Soler
Neonatal Epidemiology Unit, Hospital Cruces, University Basque Country, Bilbao, Spain

Background: CRIB score was designed to assess VLBWI initial disease severity and mortality risk. Recently, its validity has been questioned and other scales used.

Aim: To assess the validity of CRIB to predict VLBWI pre-discharge mortality cared in a single NICU during 15 years, and to compare it with other scores.

Methods: CRIB score was prospectively calculated within 24 hours of life in 1,048 inborn VLBWI admitted to a single NICU from 1994 to 2008. Scores validated by two networks Neocosur -birthweight, gestational age (GA), gender, prenatal steroid use and 1-min Apgar score-, and EuroNeoNet (adds C-Section) were calculated by regression models using the step-wise selection method. Calibration was measured by Hosmer-Lemeshow goodnessof-fit and discrimination by area under ROC curve. Comparisons between models were based on nonparametric tests over ROC curves. $\mathrm{P}<0.05$.

Results: Mean birthweight and GA were 1,120 \pm 262 $\mathrm{g}$ and $29 \pm 3$ wks. Overall neonatal and pre-discharge mortality rates were $12.4 \%$ and $15.6 \%$. The model to predict pre-discharge mortality that combined birthweight and GA (ROC: 0.862) adjusted significantly better than those based on birthweight (0.85) or GA (0.83). CRIB- adjusted model (ROC: $0.84)$ didn't improve pre-discharge mortality prediction compared to birthweight and GA model.

Both, ROC curves for Neocosur (0.868) and EuroNeoNet (0.87) scores were significantly higher than for CRIB. 